\title{
Epigallocatechin-3-gallate attenuates neointimal hyperplasia in a rat model of carotid artery injury by inhibition of high mobility group box 1 expression
}

\author{
BIN YANG ${ }^{1,2 *}$, PENG GAO $^{2 *}$, XUEJUN WU ${ }^{1}$, JIXIANG YU ${ }^{2}, \mathrm{YU} \mathrm{LI}^{2}$, \\ RANRAN MENG ${ }^{2}$, YUBIN LI ${ }^{3}$, JINGQIANG YAN ${ }^{4}$ and XING JIN ${ }^{1}$ \\ ${ }^{1}$ Department of Vascular Surgery, Shandong Provincial Hospital Affiliated to Shandong University, \\ Jinan, Shandong 250021; ${ }^{2}$ Department of Vascular Surgery, Jining First People's Hospital, Jining, \\ Shandong 272011; ${ }^{3}$ Department of Vascular Surgery, Linyi People's Hospital, Linyi, Shandong 276003; \\ ${ }^{4}$ Department of Vascular Surgery, Qingdao Municipal Hospital, Qingdao, Shandong 266011, P.R. China
}

Received June 13, 2016; Accepted April 11, 2017

DOI: 10.3892/etm.2017.4774

\begin{abstract}
Epigallocatechin-3-gallate (EGCG) is a kind of polyphenol compound, called catechin, and is extracted from green tea. EGCG has a wide range of biological activities. The present study aimed to evaluate the effect of EGCG on neointimal hyperplasia in a rat model of carotid artery balloon injury and to explore the molecular mechanisms involved. Various experiments were performed to assess the effects of EGCG on thickening of neointima, expression levels of high mobility group box 1 protein (HMGB1) and receptor of advanced glycation end products (RAGE), the inflammatory response, oxidative stress and activation of nuclear factor (NF) $-\kappa \mathrm{B}$. Results demonstrated that EGCG decreased the intimal area and the ratio of intimal area/medial area compared with the balloon injury group. The expression levels of HMGB1 and RAGE induced by balloon injury were markedly inhibited by EGCG treatment. Furthermore, the inflammatory response and oxidative stress damage, which have close correlations with HMGB1, were restrained by EGCG. Finally, EGCG treatment markedly inhibited NF- $\kappa \mathrm{B}$ activation. The present data provided evidence that EGCG attenuates neointimal hyperplasia in a model of carotid artery balloon injury, which indicated that EGCG may serve as a potential drug for restenosis in clinics.
\end{abstract}

Correspondence to: Dr Xing Jin, Department of Vascular Surgery, Shandong Provincial Hospital Affiliated to Shandong University, 324 Jingwuweiqi Road, Jinan, Shandong 250021, P.R. China

E-mail: xingjin_xj0225@163.com

*Contributed equally

Key words: epigallocatechin-3-gallate, neointimal hyperplasia, high mobility group box 1 , inflammation, oxidative stress

\section{Introduction}

Percutaneous coronary intervention (PCI) is an effective management for coronary heart disease (CHD). Restenosis, however, becomes the primary bottleneck for application of PCI (1). Increasing evidence has confirmed that neointimal proliferation following balloon dilatation and stent implantation-induced vascular injury is the primary mechanism of restenosis $(2,3)$. Thus, inhibiting vascular smooth muscle abnormal hyperplasia to prevent restenosis is a strategy for therapy of CHD. For this reason, identification of an effective clinical treatment to restrain neointimal formation is the focus of research.

High mobility group box 1 (HMGB1) belongs to the high mobility group protein family, which was identified by Goodwin and Johns (4) in 1973 and named for its high electrophoretic mobility. It has been demonstrated that HMGB1 is an important inflammatory mediator and the molecule at the heart of initiating and maintaining inflammation responses (5). The expression of HMGB1 is closely associated with the pathogenesis of sepsis (6), arthritis (7), acute pneumonia (8), hepatitis (9) and arteriosclerosis (10). Receptor of advanced glycation end products (RAGE) mediates the effects of HMGB1 (11). Furthermore, HMGB1 is reported to be expressed in intimal smooth muscle cells and injured carotid arteries tissue (12). Inhibiting HMGB1 expression may alleviate neointimal hyperplasia $(13,14)$.

Epigallocatechin-3-gallate (EGCG) is the most effective component of tea polyphenols and accounts for $45-65 \%$ of the catechinic acid content of tea (15). The metabolism products of EGCG have demonstrated strong biological activities, such as anti-inflammatory (16), antibacterial (17), anti-oxidative (18) and anti-tumor (19). Previous studies have also reported that EGCG inhibited the lipopolysaccharide (LPS)-induced inflammatory reaction by suppressing HMGB1 expression $(20,21)$. Thus, the present study speculated that EGCG may alleviate balloon-injured carotid arteries via inhibiting the overexpression of HMGB1. Based on the above evidence, the present study aimed to evaluate the inhibitory effect of 
EGCG on carotid artery balloon injury in rats and explore the related molecular mechanisms.

\section{Materials and methods}

Drug. EGCG was purchased from Meilun Biotech Co., Ltd., (Dalian, China) with purity over $95 \%$ and dissolved in normal saline prior to use. The chemical structural of EGCG was demonstrated in Fig. 1.

Establishment of a rat model of carotid artery balloon injury. Male Sprague Dawley rats (weight, 250-300 g; age, 8 weeks) were purchased from Beijing Vital River Laboratory Animal Co., Ltd., (Beijing, China). The rats of clean grade were reared at $23^{\circ} \mathrm{C}$ and $45-55 \%$ humidity, under a 12 -h light/dark cycle with access to food and water ad libitum. A total of 30 rats were randomly assigned to five groups ( $n=6$ per group). The groups were as follows: Sham operation group; carotid artery balloon injury group; injury plus EGCG (1 mg/kg); injury plus EGCG ( $2 \mathrm{mg} / \mathrm{kg})$; and injury plus EGCG ( $4 \mathrm{mg} / \mathrm{kg})$. The procedure for rat carotid artery balloon injury model establishment has been described previously (22). Briefly, rats were anesthetized with intraperitoneal injection of chloral hydrate (350 mg/kg; Sigma-Aldrich; Merck KGaA, Darmstadt, Germany). Subsequently, the skin was incised along the anterior median raphe of neck and the left carotid artery was isolated. A balloon angioplasty catheter (Edwards Lifesciences Corp, Irvine, CA, USA; diameter, $1.5 \mathrm{~mm}$; length, $20 \mathrm{~mm}$ ) was inserted through the external carotid incision to the aortic arch. The balloon was then inflated to restrict blood flow for $30 \mathrm{sec}$, and gradually withdrawn three times. Following removal of the catheter, the external carotid branch was ligated. The rats in the sham operation group underwent the same procedures without balloon catheter insertion. Penicillin $\left(2 \times 10^{5} \mathrm{U}\right.$, Taizhous Pengchange Animal Pharmaceutical Co., Ltd, Xinghua, China) was intramuscularly injected twice a day for three days to prevent infection post-operation. The rats in the EGCG treatment groups were intraperitoneally injected with 1,2 or $4 \mathrm{mg} / \mathrm{kg}$ EGCG once, $1 \mathrm{~h}$ prior to surgery and every day after surgery, respectively. Rats in the sham operation group and carotid artery balloon injury group were injected with an equal volume of normal saline at the same time points. A total of 14 days after surgery, rats were euthanized and carotid artery tissues and blood specimens were collected. All of the animal protocols were approved by and complied strictly with guidelines of the Institutional Animal Care and Use Committee of Shandong University (Jinan, China).

Histomorphometric analysis. The carotid artery tissues were fixed with $4 \%$ paraformaldehyde at $4^{\circ} \mathrm{C}$ overnight, embedded in paraffin and sectioned into $4-\mu \mathrm{m}$ thick slices. The thickening of neointima was observed by routine hematoxylin and eosin (H\&E) staining under a light microscope (Olympus Corp, Tokyo, Japan; DP73) at a magnification of $x 400$. The intimal, medial and luminal cross-sectional areas were measured. The neointima/media area $(\mathrm{I} / \mathrm{M})$ ratios were calculated.

Reverse transcription-quantitative polymerase chain reaction (RT-qPCR). Total RNA was extracted from carotid tissues using a commercial RNA extraction kit (BioTeke Biotechnology, Co.,
Ltd., Beijing, China; catalogue no. RP1201) containing DNase to remove genomic DNA. Subsequently, RNA was reverse transcribed into cDNA using M-MLV reverse transcriptase (BioTeke Biotechnology, Co., Ltd.; catalogue no. PR6502) according to the manufacturer's protocol. qPCR was performed using 2x Power Taq PCR MasterMix (BioTeke Biotechnology, Co., Ltd.; catalogue no. PR6502), according to the manufacturer's instructions. PCR amplifications were performed using the ExicyclerTM 96 (Bioneed Corp., Daejeon, Korea) as follows: $95^{\circ} \mathrm{C}$ for $10 \mathrm{~min}$ followed by 40 cycles at $95^{\circ} \mathrm{C}$ for $10 \mathrm{sec}, 60^{\circ} \mathrm{C}$ for $20 \mathrm{sec}, 72^{\circ} \mathrm{C}$ for $30 \mathrm{sec}$, and finally $4^{\circ} \mathrm{C}$ for $2 \mathrm{~min}$. Relative expression levels were calculated using the $2-^{\Delta \Delta \mathrm{Cq}}$ method (23) normalized to GAPDH expression. Specific primers used in the present study were as follows: HMGB1, forward 5'-AGG CTGACAAGGCTCGTTATG-3' and reverse 5'-TGTCAT CCGCAGCAGTGT TG-3'; RAGE, forward 5'-ACCGAGTCC GAGTCTACCA-3' and reverse 5'-GCTCTGACCGAAGCG TGA-3'; and $\beta$-actin, forward 5'-GGAGATTACTGCCCT G GCTCCTAGC-3' and reverse 5'-GGCCGGACTCATCGT ACTCCTGCTT-3'.

Western blot analysis. Western blot analysis was performed to assess the protein expression levels in carotid artery tissues. Tissue samples were lysed in lysis buffer (Wanleibio Co., Ltd., Shenyang, China) and then denatured. Equal amounts $(40 \mu \mathrm{g})$ of protein were separated by $11 \%$ SDS-PAGE and transferred to polyvinylidene difluoride membranes. Subsequent to blocking with $5 \%$ skimmed milk for $1 \mathrm{~h}$ at room temperature, the membranes were incubated with primary antibodies against HMGB1 (Boster Biological Technology, Ltd., Wuhan, China; catalogue no. BA4277; 1:400), RAGE (Boster Biological Technology, Ltd.; catalogue no. PB0530; 1:400), nuclear factor (NF)- $\kappa$ B (Boster Biological Technology, Ltd.; catalogue no. BA0610; $1: 400)$ and $\beta$-actin (Santa Cruz Biotechnology, Dallas, USA; catalogue no. sc-47778; 1:400), respectively at $4{ }^{\circ} \mathrm{C}$ overnight. After washing with $0.1 \%$ Tris buffered saline with Tween-20 three times for $5 \mathrm{~min}$, the blots were incubated with Goat Anti-Rabbit IgG secondary antibody (Wanleibio Co., Ltd.; catalogue no. WLA023; 1:5,000) for $45 \mathrm{~min}$ at $37^{\circ} \mathrm{C}$. Immunodetection was performed with an enhanced chemiluminescence detection system (Wanleibio Co., Ltd.). The optical densities of the bands relative to $\beta$-actin were quantified with a Gel Doc 2000 system (Bio-Rad Laboratories, Inc., Hercules, CA, USA).

ELISA assay. Serum inflammatory cytokine levels were determined using commercial ELISA kits: Tumor necrosis factor (TNF)- $\alpha$ (catalogue no. EK0526), interleukin (IL)-1 $\beta$ (catalogue no. EK0393), IL-6 (catalogue no. EK0412), intercellular adhesion molecule (ICAM)-1 (catalogue no. EK0372; all, BOSTER Biological Technology, Wuhan, China) and vascular cell adhesion molecule (VCAM)-1 (catalogue no. SEA547Ra; USCN Life Science Co., Ltd., Wuhan, China). The results were obtained at $450 \mathrm{~nm}$ using a microplate reader (Omega Bio-Tek, Inc., Norcross, GA, USA). The concentrations were calculated by creating a standard curve.

Malondialdehyde (MDA) determinations. MDA levels in carotid artery tissues were measured using a commercial Malondialdehyde assay kit (Nanjing Jiancheng Bioengineering 
Institute, A003-1), according to the manufacturer's instructions. Briefly, homogenized carotid artery tissues were incubated at $95^{\circ} \mathrm{C}$ for $40 \mathrm{~min}$ with thiobarbituric acid to form a new complex. The results were measured on a microplate reader (Omega Bio-Tek, Inc.) at $532 \mathrm{~nm}$ and expressed as $\mathrm{nM}$ $\mathrm{MDA} / \mathrm{mg}$ protein.

Measurement of reactive oxygen species (ROS) production. The tissue ROS levels were detected using a commercial Reactive oxygen species Assay Kit (Nanjing Jiancheng Bioengineering Institute, E004), according to the manufacturer's instructions. Briefly, the tissue homogenate was incubated with $1 \mathrm{mM}$ oxidation-sensitive fluorescent probe, DCFH-DA, for $30 \mathrm{~min}$ at $37^{\circ} \mathrm{C}$ in the dark. The samples were washed twice for $5 \mathrm{~min}$ in phosphate-buffered saline and measured on a microplate reader.

Electrophoretic mobility shift assay (EMSA). Nuclear protein was prepared from carotid artery tissues using a nuclear and cytoplasmic kit (Wanleibio Co., Ltd., Shenyang, China), according to the manufacturer's instructions. Subsequently, the protein concentration was detected using a bicinchoninic acid assay protein estimation kit (Beyotime Institute of Biotechnology, Haimen, China). NF-кB DNA binding ability was assessed by EMSA assay. Briefly, nuclear samples were incubated with a biotin end-labeled DNA probe (Viagen Biotech, Inc. Beijing, China). The DNA-protein complexes were electrophoresed on $6.5 \%$ non-denaturing polyacrylamide gels at $180 \mathrm{~V}$ for $80 \mathrm{~min}$. By electroblotting, the complexes were transferred to a positively charged nylon membrane and detected by enhanced chemiluminescence (ECL) detection system (Wanleibio Co., Ltd.). The image was obtained and optical densities of the bands were quantified by gel imaging and analysis system (Liuyi Biotechnology, Co., Ltd., Beijing, China, WD-9413B).

Statistical analysis. All results were presented as the mean \pm standard deviation. Statistical analyses were performed by one-way analysis of variance followed by Bonferroni post hoc tests using GraphPad Prism, version 5 software (GraphPad Software, Inc., La Jolla, CA, USA). P<0.05 was considered to indicate a statistically significant difference.

\section{Results}

Effect of EGCG on neointimal formation. H\&E staining assay was performed to assess the degree of neointimal formation on day 14 after balloon injury (Fig. 2). As demonstrated in Fig. 2A, there was a single layer of endothelial cells in the carotid artery wall of sham-operated rats. In the balloon injury group, non-uniform thickening of the neointima and lumen stenosis were observed. EGCG treatment markedly reduced neointima hypertrophy. The intimal area was significantly reduced in the EGCG $2 \mathrm{mg} / \mathrm{kg}(\mathrm{P}<0.05)$ and $4 \mathrm{mg} / \mathrm{kg}$ $(\mathrm{P}<0.01)$ groups compared with the injury group, and the and $\mathrm{I} / \mathrm{M}$ ratio was significantly reduced in the EGCG $4 \mathrm{mg} / \mathrm{kg}$ compared with the injury group $(\mathrm{P}<0.05$; Fig. $2 \mathrm{~B}$ and $\mathrm{E})$. A significant increase in luminal area was also observed in the EGCG $4 \mathrm{mg} / \mathrm{kg}$ group compared with the injury group $(\mathrm{P}<0.01$; Fig. 2C).

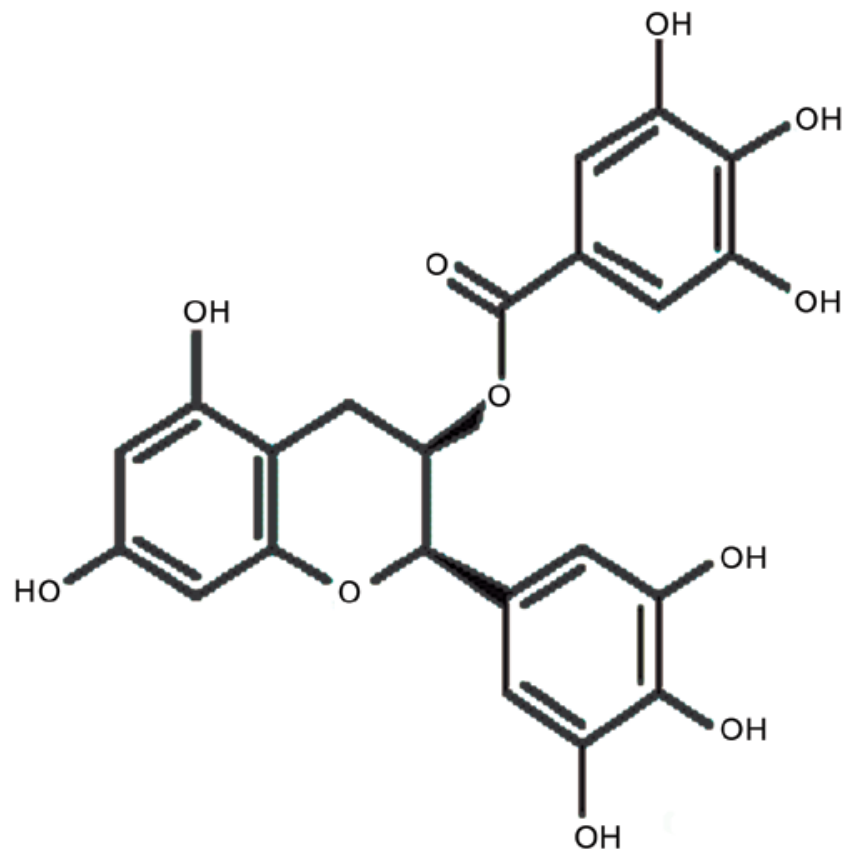

Figure 1. Chemical structure of EGCG. The molecular formula of EGCG is $\mathrm{C}_{22}$ $\mathrm{H}_{18} \mathrm{O}_{11}$ and its molecular weight is 458.38. EGCG, epigallocatechin-3-gallate.

Effect of EGCG on balloon injury-induced expression of $H M G B 1$ and its receptor, RAGE. As demonstrated in Fig. 3A and $\mathrm{B}$, compared with the sham-operated group, the mRNA expression levels of HMGB1 and RAGE were significantly increased in the balloon injury group $(\mathrm{P}<0.001)$. EGCG treatment significantly inhibited the balloon injury-induced increase in HMGB1 and RAGE mRNA expression levels in a concentration-dependent manner $(\mathrm{P}<0.05)$. Furthermore, the protein expression levels of HMGB1 and RAGE were also determined. As demonstrated in Fig. 3C and D, the levels of HMGB1 and RAGE were low in the sham-operated group, and these were significantly increased by balloon injury $(\mathrm{P}<0.001)$. EGCG treatment significantly inhibited HMGB1 and RAGE protein expression levels compared with the injury group $(\mathrm{P}<0.05)$.

Effect of EGCG on the inflammatory response. As HMGB1 is an important inflammatory mediator (5), inflammatory cytokine levels in serum were detected by ELISA. As demonstrated in Fig. 4, the production of TNF- $\alpha$, IL-1 $\beta$, IL-6, ICAM-1 and VCAM-1 were significantly increased by balloon injury compared with the sham-operated group $(\mathrm{P}<0.001)$, while treatment with EGCG significantly suppressed the excess production of these inflammatory cytokines $(\mathrm{P}<0.05)$.

Antioxidant effect of EGCG in rats undergoing carotid artery balloon injury. Furthermore, the oxidative stress markers, MDA and ROS, were also assessed. As demonstrated in Fig. 5, the levels of MDA and ROS were significantly increased in the balloon injury group compared with the sham-operated group $(\mathrm{P}<0.001)$. Following treatment with different concentrations of EGCG, the levels of MDA and ROS were significantly decreased compared with the balloon injury group $(\mathrm{P}<0.05)$.

Effect of EGCG on $N F-\kappa B$ activation. To further clarify the molecular mechanism of EGCG on alleviating carotid artery 
A

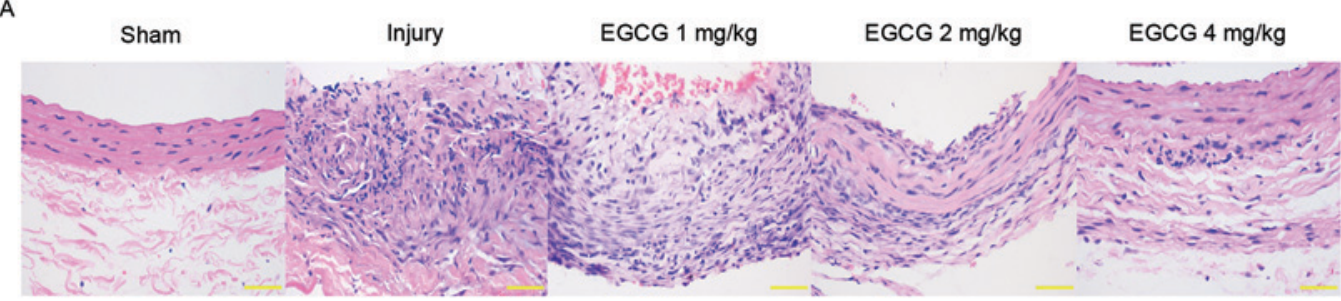

B

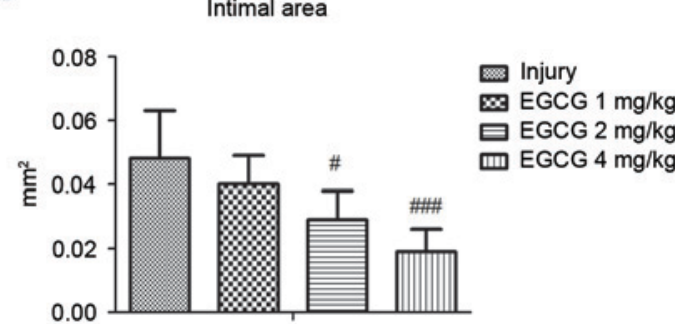

D

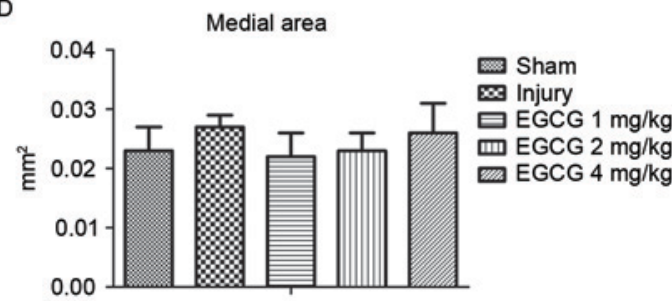

C

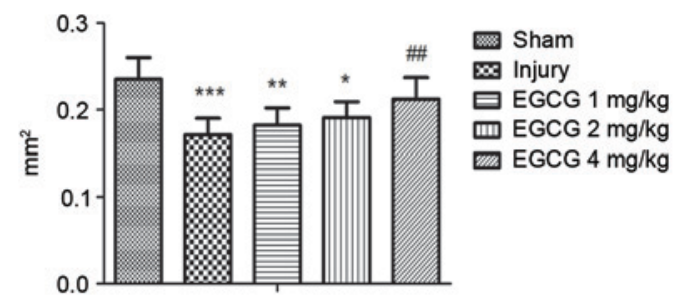

E

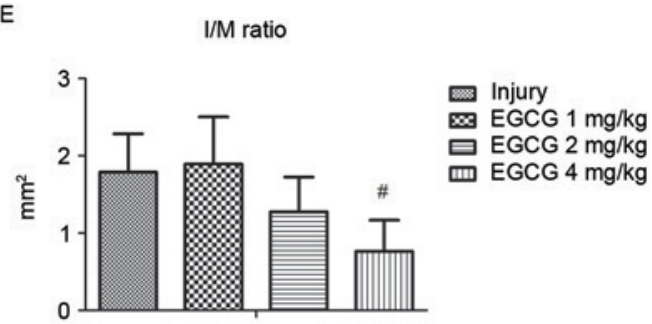

Figure 2. EGCG attenuates neointimal hyperplasia induced by carotid artery balloon injury. (A) Sections of carotid artery tissues 14 days after balloon injury were stained with hematoxylin and eosin (magnification, $\mathrm{x} 400$; scale bar, $50 \mu \mathrm{m}$ ). The areas of (B) intima, (C) lumen and (D) media were shown and the (E) I/M ratios were calculated. Data are presented as the mean + standard deviation $(\mathrm{n}=6) .{ }^{*} \mathrm{P}<0.05,{ }^{* *} \mathrm{P}<0.01$ and ${ }^{* * *} \mathrm{P}<0.001 \mathrm{vs}$. the sham group; ${ }^{\#} \mathrm{P}<0.05$, ${ }^{\# \#} \mathrm{P}<0.01$ and

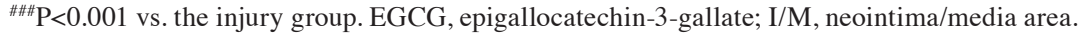

balloon injury in rats, the effects of EGCG on the expression and activity of NF- $\kappa \mathrm{B}$ was evaluated. As demonstrated in Fig. 6A, the expression of $\mathrm{NF}-\kappa \mathrm{B}$ in the nucleus was significantly increased, while cytosolic $\mathrm{NF}-\kappa \mathrm{B}$ was significantly decreased in the balloon injury group compared with the sham-operated group $(\mathrm{P}<0.001)$. Treatment with EGCG significantly inhibited the translocation of NF- $\kappa \mathrm{B}$ from cytosol to nucleus induced by balloon injury $(\mathrm{P}<0.05)$. Furthermore, the activity of $\mathrm{NF}-\kappa \mathrm{B}$ binding to DNA was assessed by EMSA assay. As demonstrated in Fig. $6 \mathrm{~B}$, the activity of $\mathrm{NF}-\kappa \mathrm{B}$ in the balloon injury group was enhanced significantly compared with the sham-operated group $(\mathrm{P}<0.001)$, while EGCG dose-dependently inhibited the activation of NF- $\mathrm{NB}$ significantly compared with the balloon injury group $(\mathrm{P}<0.05)$.

\section{Discussion}

Although PCI has been widely used for treating vascular stenosis, restenosis after PCI has always been a difficult problem to overcome in therapy of CHD (1). Currently, the optimal treatment has not been demonstrated in clinics. Therefore, more effective treatments are required. EGCG, extracted from Chinese green tea, is used in a vast range of foods, pharmaceuticals, cosmetics and health products $(24,25)$. The present study aimed to observe the effect of EGCG on intima thickening and investigate the related molecular mechanisms. According to the results of the present study, EGCG effectively ameliorated the morphological changes in the carotid artery following balloon injury, decreased the neointimal area and I/M ratio, increased luminal area, and demonstrated a significant effect against intimal hyperplasia and restenosis of vessels.

HMGB1, an inflammatory cytokine, belongs to the alarmin family and has an important role in the progression of inflammation (26). Growing evidence has demonstrated that HMGB1 was involved in a variety of inflammatory diseases (27-29). Furthermore, HMGB1 has been indicated to be a powerful regulator of balloon injury-induced neointimal hyperplasia (14). Research has also demonstrated that plasma HMGB1 levels may serve as a prognostic biomarker in patients with ST-segment elevation myocardial infarction treated with PCI (30). RAGE, which is not highly expressed in normal tissues, was the first identified receptor of HMGB1 and is highly expressed when combined with its ligand (31). As the present study indicated that balloon injury-induced expression of HMGB1 and RAGE were significantly inhibited by EGCG, we probed the possibility that the suppression of the inflammatory response is related to the downregulation of HMGB1. The role of the inflammatory response in restenosis has attracted increased attention. A study by Libby et al (32) demonstrated that the release of inflammatory mediators facilitates neointimal formation and restenosis of angioplasty. Research has also provided evidence that leukocytes were persistently activated following aorta balloon injury in rabbits (33). HMGB1 is also reported to have cross-links with proinflammatory cytokines, such as TNF- $\alpha$, IL-1 $\beta$ and IL-6, which amplify the inflammation $(34,35)$. According to 
A

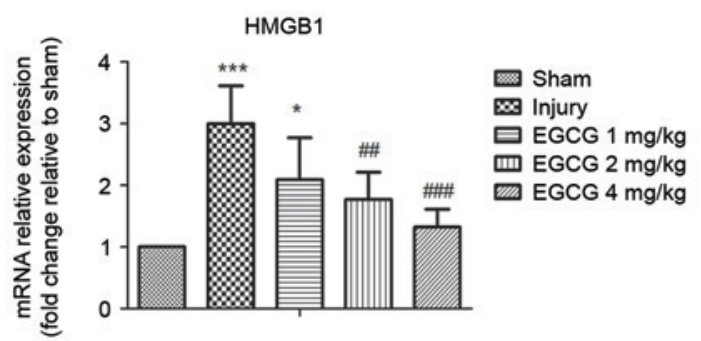

c
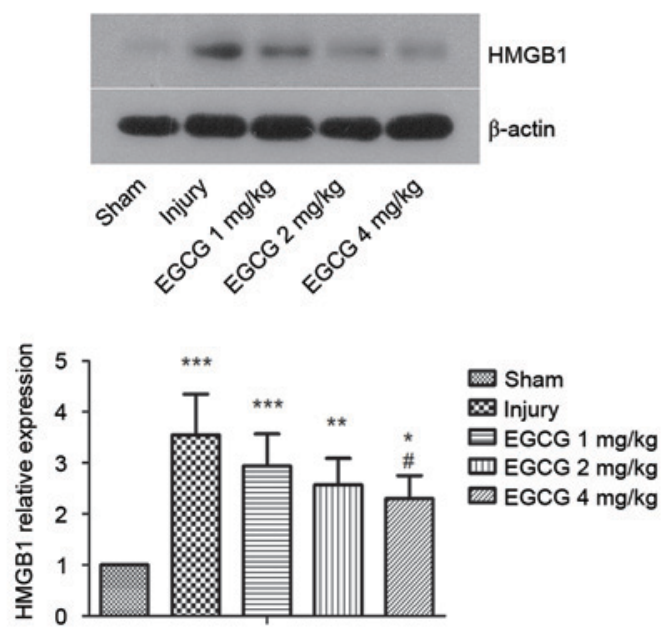
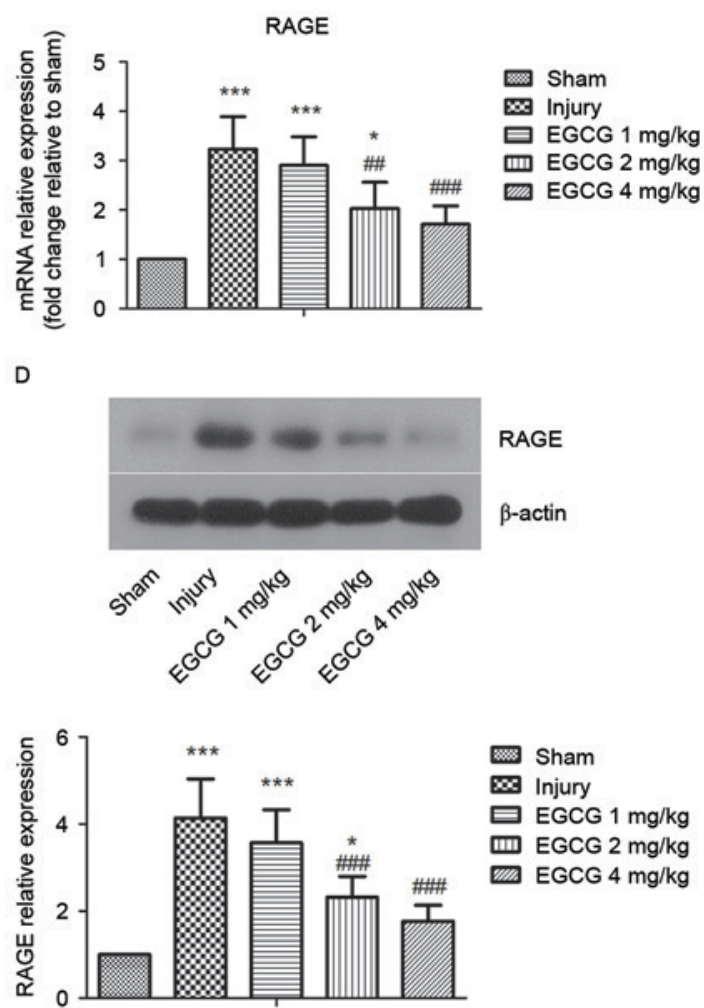

Figure 3. EGCG inhibits balloon injury-induced HMGB1 and RAGE expression levels. mRNA expression levels of (A) HMGB1 and (B) RAGE in artery tissues were determined by reverse transcription-quantitative polymerase chain reaction. Protein expression levels of (C) HMGB1 and (D) RAGE in artery tissues were detected by western blotting. $\beta$-actin was used as a loading control. The protein bands were quantified by gray scanning. Data are presented as the mean + standard deviation $(\mathrm{n}=6) .{ }^{*} \mathrm{P}<0.05,{ }^{* * *} \mathrm{P}<0.01$ and ${ }^{* * * *} \mathrm{P}<0.001$ vs. the sham group; ${ }^{\#} \mathrm{P}<0.05,{ }^{\# \#} \mathrm{P}<0.01$ and ${ }^{\# \# \#} \mathrm{P}<0.001$ vs. the injury group. EGCG, epigallocatechin-3-gallate; HMGB1, high mobility group box 1; RAGE, receptor of advanced glycation end products.

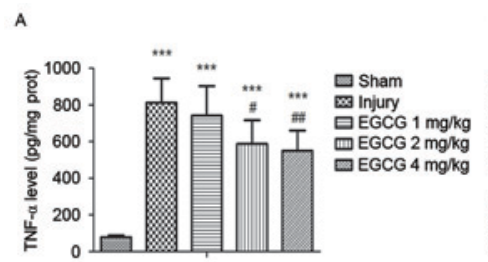

\section{B}
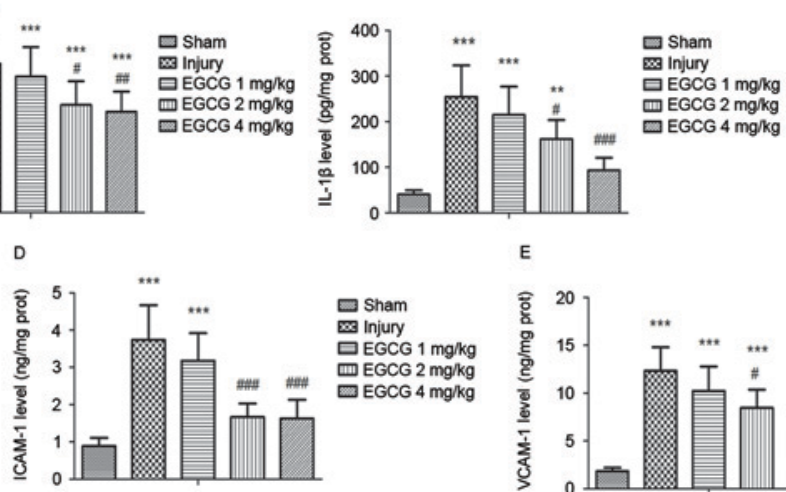

$c$

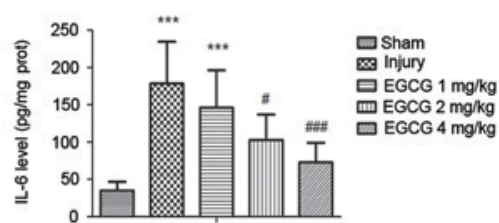

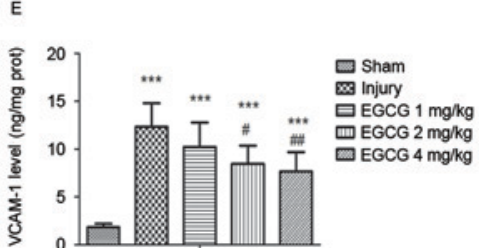

Figure 4. EGCG inhibits the balloon injury-induced inflammatory response. (A) TNF- $\alpha$, (B) IL-1 $\beta$, (C) IL-6, (D) ICAM-1 and (E) VCAM-1 levels in peripheral blood were detected by ELISA. Three independent experiments were performed. Data are presented as the mean + standard deviation $(\mathrm{n}=6)$. ${ }^{* *} \mathrm{P}<0.01$ and ${ }^{* * * *} \mathrm{P}<0.001$ vs. the sham group; ${ }^{\#} \mathrm{P}<0.05$, ${ }^{\# \#} \mathrm{P}<0.01$ and ${ }^{\# \# \#} \mathrm{P}<0.001$ vs. the injury group. EGCG, epigallocatechin-3-gallate; TNF- $\alpha$, tumor necrosis factor $\alpha$; IL, interleukin; ICAM, intercellular adhesion molecule; VCAM, vascular cell adhesion molecule; prot, protein.

the present study, the effect of EGCG against arterial balloon injury should be related to its suppression of inflammation.

Prior studies have suggested that oxidative stress is closely related to restenosis following balloon injury $(36,37)$. Oxidative stress is the key link of the development of atherosclerosis (38). A large number of ROS were produced in damaged endothelial cells, which induces endothelium dysfunction and vascular smooth muscle cell proliferation (39). As the metabolite of oxidative stress, MDA may indirectly reflect the degree of vascular injury. Increasing evidence has reported that EGCG, as a powerful anti-oxidant, has pivotal roles in anti-cancer and anti-vascular disease (40-42). The results of the present study were consistent with previous studies and demonstrated that EGCG significantly inhibited oxidative stress induced by balloon injury by decreasing levels of MDA and ROS. Oxidative stress is always closely related to 

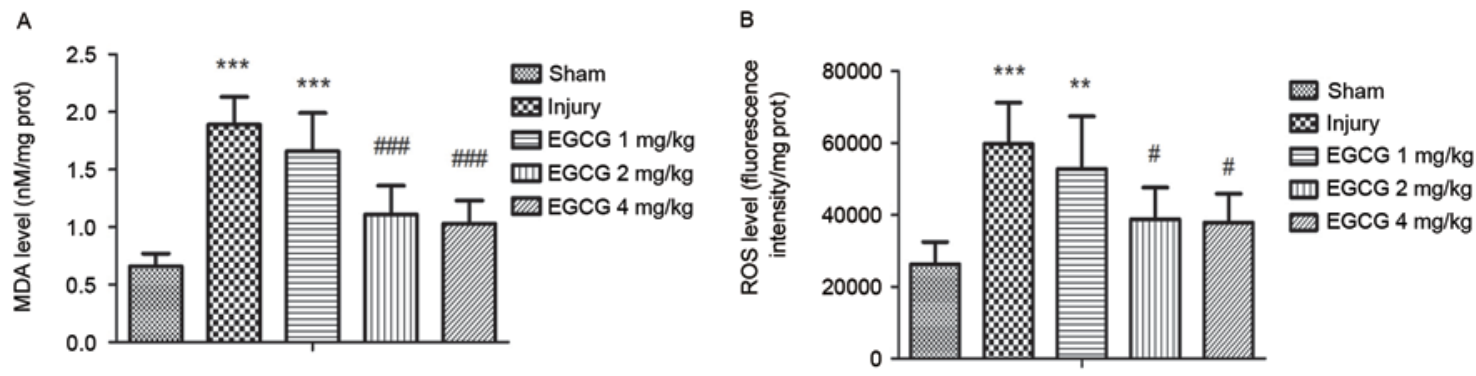

Figure 5. EGCG inhibits balloon injury-induced oxidative stress damage. (A) MDA and (B) ROS levels were assessed. Three independent experiments were performed. Data are presented as the mean + standard deviation $(n=6) .{ }^{* *} \mathrm{P}<0.01$ and ${ }^{* * * *} \mathrm{P}<0.001$ vs. the sham group; ${ }^{\#} \mathrm{P}<0.05$ and ${ }^{\# \# \#} \mathrm{P}<0.001$ vs. the injury group. EGCG, epigallocatechin-3-gallate; MDA, malondialdehyde; ROS, reactive oxygen species; prot, protein.
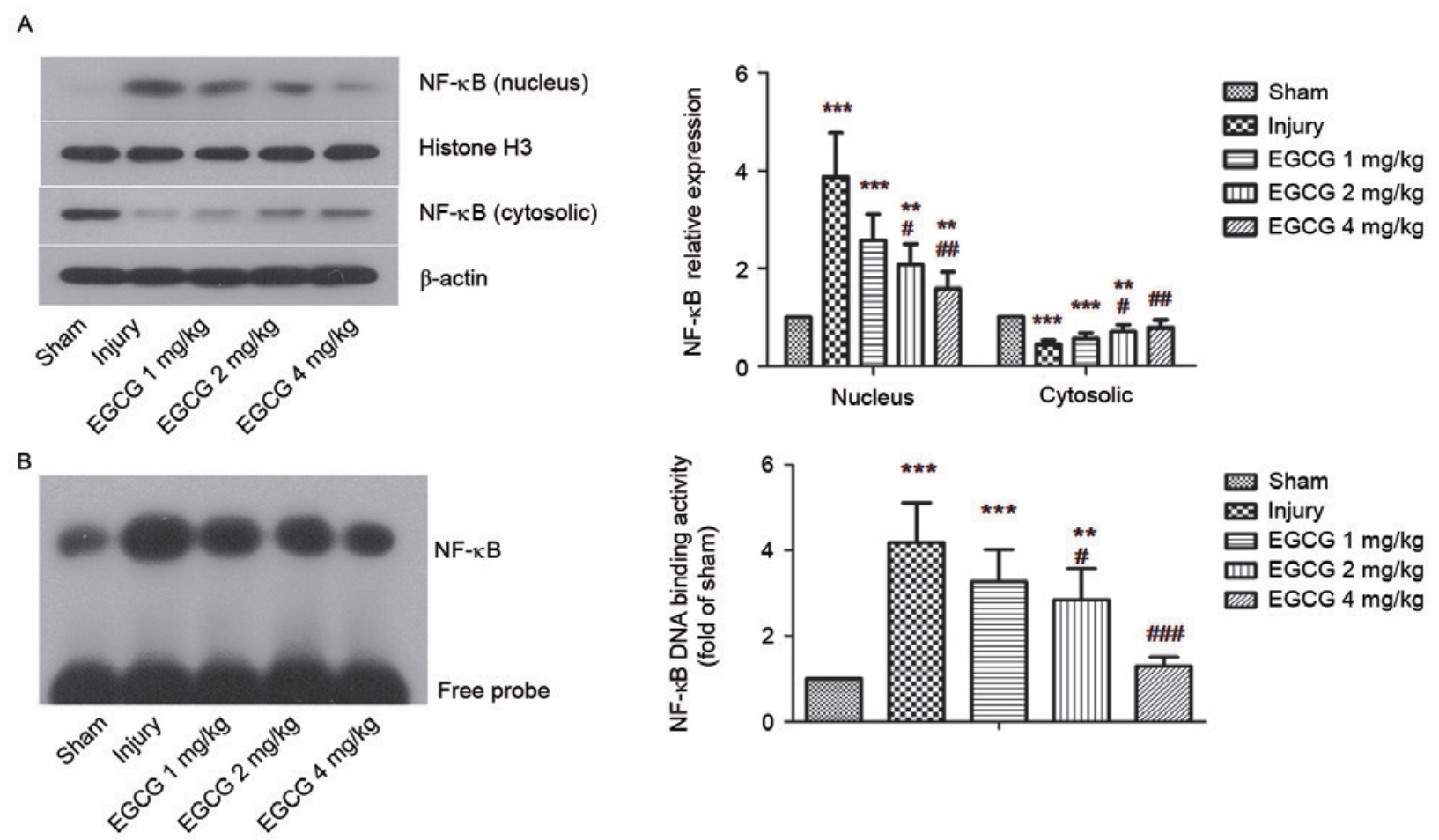

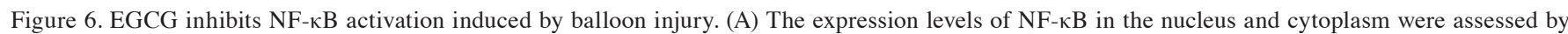
western blot assay. Histone $\mathrm{H} 3$ and $\beta$-actin were used as loading controls. The protein bands were quantified by gray scanning (n=6). (B) Electrophoretic mobility shift assay was performed to assess the DNA binding activity of NF- $\mathrm{kB}$. Three independent experiments were performed. Data are presented as the mean + standard deviation $(\mathrm{n}=6) .{ }^{* *} \mathrm{P}<0.01$ and ${ }^{* * *} \mathrm{P}<0.001$ vs. the sham group; ${ }^{*} \mathrm{P}<0.05,{ }^{\# \#} \mathrm{P}<0.01$ and ${ }^{\# \# \#} \mathrm{P}<0.001$ vs. the injury group. EGCG, epigallocatechin-3-gallate; NF, nuclear factor.

inflammation (43). Previous research has indicated that use of antioxidants may protect against inflammatory diseases (44). A previous study also demonstrated that there is a mutual enhancement between HMGB1 and oxidative stress (45). These results indicate that the inhibition of oxidative stress may suppress HMGB1 expression. In the present study, the results suggested that EGCG was able to restrain the oxidative stress induced by balloon injury to inhibit HMGB1 expression, which suppressed the inflammatory response and attenuated neointimal hyperplasia in a rat model of carotid artery injury.

$\mathrm{NF}-\mathrm{KB}$ is an important transcription factor that is activated by nuclear translocation and induces the overexpression of multiple cytokines, including TNF- $\alpha$, IL- $1 \beta$ and IL- 6 , and adhesion molecules, such as ICAM-1 and VCAM-1 $(46,47)$. Thus, it has a central role in the inflammatory response $(46,47)$.
Research has demonstrated that HMGB1 was induced by $\mathrm{NF}-\mathrm{\kappa B}$ activation, thus promoting the progression of acute lung injury (48). A study by Tan et al (49) indicated that suppression of NF- $\mathrm{KB}$ was able to reduce LPS-induced HMGB1. Therefore, inhibiting the activation of NF- $\mathrm{\kappa B}$ may downregulate the level of HMGB1 to attenuate carotid artery injury. The findings of the present study demonstrated that EGCG significantly inhibited the activation of $\mathrm{NF}-\mathrm{\kappa B}$, which subsequently reduced HMGB1 expression.

In conclusion, the present study provides evidence that EGCG effectively attenuates neointimal hyperplasia induced by carotid artery injury. Inhibition of HMGB1 expression, suppression of the inflammatory reaction and attenuation of oxidative stress were involved in the protective effects of EGCG. Therefore, EGCG may be a potential treatment for restenosis following PCI. 


\section{References}

1. Liu J, Li M, Lu H, Qiao W, Xi D, Luo T, Xiong H and Guo Z: Effects of probucol on restenosis after percutaneous coronary intervention: A systematic review and meta-analysis. PLoS One 10: e0124021, 2015.

2. Costa MA and Simon DI: Molecular basis of restenosis and drug-eluting stents. Circulation 111: 2257-2273, 2005.

3. Inoue $\mathrm{T}$ and Node $\mathrm{K}$ : Molecular basis of restenosis and novel issues of drug-eluting stents. Circ J 73: 615-621, 2009.

4. Goodwin GH and Johns EW: Isolation and characterisation of two calf-thymus chromatin non-histone proteins with high contents of acidic and basic amino acids. Eur J Biochem 40: 215-219, 1973

5. Kim TH, Ku SK and Bae JS: Inhibitory effects of kaempferol-3-O-sophoroside on HMGB1-mediated proinflammatory responses. Food Chem Toxicol 50: 1118-1123, 2012.

6. Fang WH, Yao YM, Shi ZG, Yu Y, Wu Y, Lu LR and Sheng ZY: The significance of changes in high mobility group-1 protein mRNA expression in rats after thermal injury. Shock 17: 329-333, 2002

7. Park SY, Lee SW, Kim HY, Lee WS, Hong KW and Kim CD: HMGB1 induces angiogenesis in rheumatoid arthritis via HIF-1o activation. Eur J Immunol 45: 1216-1227, 2015.

8. Abraham E, Arcaroli J, Carmody A, Wang H and Tracey KJ HMG-1 as a mediator of acute lung inflammation. J Immunol 165 : 2950-2954, 2000.

9. Li X, Liu HC, Yao QY, Xu BL, Zhang SC and Tu CT: Quercetin protects mice from ConA-induced hepatitis by inhibiting HMGB1-TLR expression and down-regulating the nuclear factor Kappa B pathway. Inflammation 39: 96-106, 2016.

10. Umahara T, Uchihara T, Koyama S, Hashimoto T, Akimoto J, Haraoka J and Iwamoto T: Local extension of HMGB1 in atherosclerotic lesions of human main cerebral and carotid arteries Histol Histopathol 29: 235-242, 2014.

11. Kokkola R, Andersson A, Mullins G, Ostberg T, Treutiger CJ Arnold B, Nawroth P, Andersson U, Harris RA and Harris HE: RAGE is the major receptor for the proinflammatory activity of HMGB1 in rodent macrophages. Scand J Immunol 61: 1-9, 2005.

12. Cai J, Yuan H, Wang Q, Yang H, Al-Abed Y, Hua Z, Wang J, Chen $\mathrm{D}, \mathrm{Wu} \mathrm{J}, \mathrm{Lu} \mathrm{B}$, et al: HMGB1-driven inflammation and intimal hyperplasia after arterial injury involves cell-specific actions mediated by TLR4. Arterioscler Thromb Vasc Biol 35: 2579-2593, 2015

13. Kalinina N, Agrotis A, Antropova Y, DiVitto G, Kanellakis P, Kostolias G, Ilyinskaya O, Tararak E and Bobik A: Increased expression of the DNA-binding cytokine HMGB1 in human atherosclerotic lesions: Role of activated macrophages and cytokines. Arterioscler Thromb Vasc Biol 24: 2320-2325, 2004.

14. Chen J, Zhang J, Xu L, Xu C, Chen S, Yang J and Jiang H: Inhibition of neointimal hyperplasia in the rat carotid artery injury model by a HMGB1 inhibitor. Atherosclerosis 224 332-339, 2012

15. Tanaka T, Matsuo $Y$ and Kouno I: A novel black tea pigment and two new oxidation products of epigallocatechin-3-O-gallate. J Agric Food Chem 53: 7571-7578, 2005.

16. Yun M, Seo G, Lee JY, Chae GT and Lee SB: Epigallocatechin-3-gallate attenuates the AIM2-induced secretion of IL-1 $\beta$ in human epidermal keratinocytes. Biochem Biophys Res Commun 467: 723-729, 2015.

17. Hu J, Du X, Huang C, Fu D, Ouyang X and Wang Y: Antibacterial and physical properties of EGCG-containing glass ionomer cements. J Dent 41: 927-934, 2013.

18. Oršolić N, Sirovina D, Gajski G, Garaj-Vrhovac V, Jazvinšćak Jembrek M and Kosalec I: Assessment of DNA damage and lipid peroxidation in diabetic mice: Effects of propolis and epigallocatechin gallate (EGCG). Mutat Res 757: 36-44, 2013.

19. Mun ST, Bae DH and Ahn WS: Epigallocatechin gallate with photodynamic therapy enhances anti-tumor effects in vivo and in vitro. Photodiagnosis Photodyn Ther 11: 141-147, 2014

20. Li W, Ashok M, Li J, Yang H, Sama AE and Wang H: A major ingredient of green tea rescues mice from lethal sepsis partly by inhibiting HMGB1. PLoS One 2: e1153, 2007.

21. Li W, Zhu S, Li J, Assa A, Jundoria A, Xu J, Fan S, Eissa NT, Tracey KJ, Sama AE and Wang H: EGCG stimulates autophagy and reduces cytoplasmic HMGB1 levels in endotoxin-stimulated macrophages. Biochem Pharmacol 81: 1152-1163, 2011.

22. Zhang W and Trebak M: Vascular balloon injury and intraluminal administration in rat carotid artery. J Vis Exp: Dec 23, 2014 doi: 10.3791/52045.
23. Livak KJ and Schmittgen TD: Analysis of relative gene expression data using real-time quantitative PCR and the 2(-Delta Delta C(T)) Method. Methods 25: 402-408, 2001.

24. Katiyar SK and Elmets CA: Green tea polyphenolic antioxidants and skin photoprotection (Review). Int J Oncol 18: 1307-1313, 2001.

25. Moderno PM, Carvalho M and Silva BM: Recent patents on Camellia sinensis: Source of health promoting compounds. Recent Pat Food Nutr Agric 1: 182-192, 2009.

26. Wu X, Mi Y, Yang H, Hu A, Zhang Q and Shang C: The activation of HMGB1 as a progression factor on inflammation response in normal human bronchial epithelial cells through RAGE/JNK/NF-kB pathway. Mol Cell Biochem 380: 249-257, 2013.

27. Banerjee S, Friggeri A, Liu G and Abraham E: The C-terminal acidic tail is responsible for the inhibitory effects of HMGB1 on efferocytosis. J Leukoc Biol 88: 973-979, 2010.

28. Winter N, Meyer A, Richter A, Krisponeit D and Bullerdiek J: Elevated levels of HMGB1 in cancerous and inflammatory effusions. Anticancer Res 29: 5013-5017, 2009.

29. Dave SH, Tilstra JS, Matsuoka K, Li F, DeMarco RA, Beer-Stolz D, Sepulveda AR, Fink MP, Lotze MT and Plevy SE: Ethyl pyruvate decreases HMGB1 release and ameliorates murine colitis. J Leukoc Biol 86: 633-643, 2009.

30. Sorensen MV, Pedersen S, Møgelvang R, Skov-Jensen J and Flyvbjerg A: Plasma high-mobility group box 1 levels predict mortality after ST-segment elevation myocardial infarction. JACC Cardiovasc Interv 4: 281-286, 2011.

31. Weber DJ, Allette YM, Wilkes DS and White FA: The HMGB1-RAGE inflammatory pathway: Implications for brain injury-induced pulmonary dysfunction. Antioxid Redox Signal 23: 1316-1328, 2015.

32. Libby P, Schwartz D, Brogi E, Tanaka H and Clinton SK: A cascade model for restenosis. A special case of atherosclerosis progression. Circulation 86 (Suppl 6): III47-III52, 1992.

33. Tanaka H, Sukhova GK, Swanson SJ, Clinton SK, Ganz P, Cybulsky MI and Libby P: Sustained activation of vascular cells and leukocytes in the rabbit aorta after balloon injury. Circulation 88: 1788-1803, 1993.

34. Andersson U, Wang H, Palmblad K, Aveberger AC, Bloom O, Erlandsson-Harris H, Janson A, Kokkola R, Zhang M, Yang H and Tracey KJ: High mobility group 1 protein (HMG-1) stimulates proinflammatory cytokine synthesis in human monocytes. J Exp Med 192: 565-570, 2000.

35. El Gazzar M, Yoza BK, Chen X, Garcia BA, Young NL and McCall CE: Chromatin-specific remodeling by HMGB1 and linker histone $\mathrm{H} 1$ silences proinflammatory genes during endotoxin tolerance. Mol Cell Biol 29: 1959-1971, 2009.

36. Kawamoto R, Yamashita A, Nishihira K, Furukoji E, Hatakeyama K, Ishikawa T, Imamura T, Itabe H, Eto T and Asada Y: Different inflammatory response and oxidative stress in neointimal hyperplasia after balloon angioplasty and stent implantation in cholesterol-fed rabbits. Pathol Res Pract 202: 447-456, 2006

37. Murthy SN, Desouza CV, Bost NW, Hilaire RC, Casey DB Badejo AM, Dhaliwal JS, McGee J, McNamara DB, Kadowitz PJ and Fonseca VA: Effects of salsalate therapy on recovery from vascular injury in female Zucker fatty rats. Diabetes 59: 3240-3246, 2010

38. Şerban $C$ and Drăgan S: The relationship between inflammatory and oxidative stress biomarkers, atherosclerosis and rheumatic diseases. Curr Pharm Des 20: 585-600, 2014.

39. $\mathrm{Wu} \mathrm{X}$ and $\mathrm{Lu} \mathrm{Q}$ : Expression and significance of $\alpha$-SMA and PCNA in the vascular adventitia of balloon-injured rat aorta. Exp Ther Med 5: 1671-1676, 2013

40. Zapf MA, Kothari AN, Weber CE, Arffa ML, Wai PY, Driver J, Gupta GN, Kuo PC and Mi Z: Green tea component epigallocatechin-3-gallate decreases expression of osteopontin via a decrease in mRNA half-life in cell lines of metastatic hepatocellular carcinoma. Surgery 158: 1039-1048, 2015.

41. Afzal M,Safer AM and Menon M: Green tea polyphenols and their potential role in health and disease. Inflammopharmacology 23: 151-161, 2015.

42. Shu Z, Yu M, Zeng G, Zhang $X, W u ~ L$ and Tan $X$ : Epigallocatechin-3-gallate inhibits proliferation of human aortic smooth muscle cells via up-regulating expression of mitofusin 2 . Eur J Cell Biol 93: 137-144, 2014.

43. El Assar M, Angulo J and Rodriguez-Mañas L: Oxidative stress and vascular inflammation in aging. Free Radic Biol Med 65: 380-401, 2013 
44. Vitali R, Palone F, Pierdomenico M, Negroni A, Cucchiara S, Aloi M, Oliva S and Stronati L: Dipotassium glycyrrhizate via HMGB1 or AMPK signaling suppresses oxidative stress during intestinal inflammation. Biochem Pharmacol 97: 292-299, 2015.

45. Mohammad G, Alam K, Nawaz MI, Siddiquei MM, Mousa A and Abu El-Asrar AM: Mutual enhancement between high-mobility group box-1 and NADPH oxidase-derived reactive oxygen species mediates diabetes-induced upregulation of retinal apoptotic markers. J Physiol Biochem 71: 359-372, 2015.

46. Chen GL, Zhang JJ, Kao X, Wei LW and Liu ZY: Emodin ameliorates lipopolysaccharides-induced corneal inflammation in rats. Int J Ophthalmol 8: 665-669, 2015.
47. Wang YS, Li YX, Zhao P, Wang HB, Zhou R, Hao YJ, Wang J, Wang SJ, Du J, Ma L, et al: Anti-inflammation effects of oxysophoridine on cerebral ischemia-reperfusion injury in mice. Inflammation 38: 2259-2268, 2015.

48. Ni YF, Tian F, Lu ZF, Yang GD, Fu HY, Wang J, Yan XL, Zhao YC, Wang YJ and Jiang T: Protective effect of nicotine on lipopolysaccharide-induced acute lung injury in mice. Respiration 81: 39-46, 2011

49. Tan Y, Wang Q, She Y, Bi X and Zhao B: Ketamine reduces LPS-induced HMGB1 via activation of the Nrf2/HO-1 pathway and NF-kB suppression. J Trauma Acute Care Surg 78: 784-792, 2015. 